\title{
Imaging, Pathology, and Immune Correlates in the Woodchuck Hepatic Tumor Model
}

This article was published in the following Dove Press journal:

Journal of Hepatocellular Carcinoma

\author{
Michal Mauda-Havakuk, ' \\ Andrew S Mikhail,' \\ Matthew F Starost, 2 \\ Elizabeth C Jones, ${ }^{3}$ Baktiar Karim, ${ }^{4}$ \\ David E Kleiner, ${ }^{5}$ Ari Partanen, (D) \\ Juan A Esparza-Trujillo, (D) \\ Ivane Bakhutashvili,' \\ Paul G Wakim, ${ }^{6}$ Michael T Kassin, ' \\ Andrew L Lewis, ${ }^{7}$ \\ John W Karanian,' \\ Bradford J Wood, (iD) 8 \\ William F Pritchard' \\ 'Center for Interventional Oncology, \\ Radiology and Imaging Sciences, Clinical \\ Center, National Institutes of Health, \\ Bethesda, MD, USA; ${ }^{2}$ Division of Veterinary \\ Resources, National Institutes of Health, \\ Bethesda, MD, USA; ${ }^{3}$ Radiology and \\ Imaging Sciences, Clinical Center, National \\ Institutes of Health, Bethesda, MD, USA; \\ ${ }^{4}$ National Cancer Institute, National \\ Institutes of Health, Frederick, MD, USA \\ ${ }^{5}$ Center for Cancer Research, Clinical \\ Center, National Cancer Institute, \\ Bethesda, MD, USA; ${ }^{6}$ Biostatistics and \\ Clinical Epidemiology Service, National \\ Institutes of Health Clinical Center, \\ Bethesda, MD, USA; ${ }^{7}$ Biocompatibles UK \\ Ltd (a BTG International Group Company), \\ Camberley, UK; ${ }^{8}$ Center for Interventional \\ Oncology, Radiology and Imaging Sciences, \\ Clinical Center, National Institute of \\ Biomedical Imaging and Bioengineering and \\ National Cancer Institute Center for \\ Cancer Research, National Institutes of \\ Health, Bethesda, MD, USA
}

Correspondence: William F Pritchard Center for Interventional Oncology, Radiology and Imaging Sciences, Clinical Center, National Institutes of Health, 10 Center Drive, Room 3N320B, MSC II 82, Bethesda, MD, 20892, USA

Tel + I 240-760-0I53

Fax + I 30I-496-9933

Email william.pritchard@nih.gov
Background: Woodchucks chronically infected with woodchuck hepatitis virus (WHV), which resembles human hepatitis B virus, develop spontaneous hepatic tumors and may be an important biological and immunological model for human HCC. Nonetheless, this model requires further validation to fully realize its translational potential.

Methods: Woodchucks infected at birth with WHV that had developed HCC $(n=12)$ were studied. Computed tomography, ultrasound, and magnetic resonance imaging were performed under anesthesia. LI-RADS scoring and correlative histologic analysis of sectioned tissues were performed. For immune characterization of tumors, CD3 (T cells), CD4 (T helpers), NCAM (Natural killers), FOXP3 (T-regulatory), PDL-1 (inhibitory checkpoint protein), and the human hepatocellular carcinoma (HCC) biomarker alpha-fetoprotein (AFP) immunohistochemical stains were performed.

Results: Forty tumors were identified on imaging of which 29 were confirmed to be HCC with 26 categorized as LR-4 or 5. The remainder of the tumors had benign histology including basophilic foci, adenoma, and lipidosis as well as pre-malignant dysplastic foci. LR-4 and LR-5 lesions showed high sensitivity (90\%) and specificity (100\%) for malignant and pre-malignant tumors. Natural killers count was found to be 2-5 times lower in tumors relative to normal parenchyma while other immune cells were located in the periphery of tumors. Tumors expressed AFP and did not express PD-L1.

Conclusion: Woodchucks chronically infected with WHV developed diverse hepatic tumor types with diagnostic imaging, pathology, and immune patterns comparable to that in humans. This unique animal model may provide a valuable tool for translation and validation of novel image-guided and immune-therapeutic investigations.

Keywords: carcinoma, hepatocellular, radiology, interventional oncology, immunotherapy, models, animal, tumor microenvironment

\section{Introduction}

Diagnosis of hepatocellular carcinoma (HCC) in at-risk populations is performed mainly with imaging. The Liver Imaging Reporting and Data System (LI-RADS) was designed to standardize the interpretation of imaging for at-risk patients. Imaging features are used to categorize tumors as either having intermediate (LR-3), high (LR-4), or definite (LR-5) probability for malignancy. ${ }^{1}$ When typical features defined by LI-RADS are observed, a diagnosis may be made without the need for biopsy. ${ }^{2}$ Pathologic confirmation of HCC with tissue sampling and genomic analyses has improved our understating of the molecular pathogenesis of HCC. However, since biopsy is not routine worldwide for definite HCC (LR-5), the resulting paucity of available tissue samples limits the assessment of HCC carcinogenesis and places the 
development of novel targeted therapies at a disadvantage compared with most cancers. $^{3}$

Locoregional approaches such as ablation and intra-arterial therapies are standard treatments with curative and palliative intent, respectively. However, more than $80 \%$ of HCC patients are diagnosed at advanced stages for which local, curative therapies are not an option and 5-year survival rates are only $18 \%{ }^{4}$

In 2007, sorafenib became the first drug approved by the Food and Drug Administration for first-line systemic therapy of HCC despite offering only a three-month improvement in survival compared to placebo. ${ }^{5}$ In 2017 nivolumab, a PD-1 checkpoint inhibitor, was approved for patients who have been previously treated with sorafenib after achieving 14-20\% overall response. ${ }^{6}$ Most recently, FDA approval was granted to the combination therapy Atezolizumab plus Bevacizumab for patients with unresectable HCC after achieving prolonged overall survival compared to sorafenib. ${ }^{7}$ Immunotherapy holds great promise in HCC therapy, yet there remains an urgent need for curative treatments for the majority of HCC patients.

Animal models that mimic human HCC are needed to expedite the translation of new treatments into the clinic. These preclinical models should ideally reflect the complexity and diversity of human hepatic pathology, as well as recapitulate genetic, immune, and microenvironmental features of human hepatic tumors. Most of our understanding of the mechanisms of the immune system in liver cancer has been drawn from experiments in murine models. However, researchers are limited in their ability to model and apply locoregional techniques in mice due to geometric constraints imposed by their small anatomy relative to the clinical devices. Mouse models also fall short in capturing human tumor molecular heterogeneity, and they do not fully replicate the human immune system. ${ }^{8}$ Woodchucks are the largest HCC-bearing animal model to date, allowing researchers to perform locoregional therapies using commercially available devices used in humans. ${ }^{9}$ Furthermore, the woodchuck HCC model may more closely resemble human HCC. Woodchucks infected as newborns with woodchuck hepatitis virus (WHV) spontaneously develop hepatic tumors. ${ }^{10}$ WHV infection mimics virologic and immunological responses observed in human HBV infection. ${ }^{11}$ This model has been used for the pre-clinical evaluation of efficacy and safety of antiviral compounds as well as antitumor compounds for prevention and treatment of HCC. ${ }^{12,13}$
Herein we aim to define imaging features of hepatic tumors in woodchucks using clinical imaging modalities and LI-RADS classification and identify corresponding histopathologic features and tumor immune patterns. The ultimate goal of this study was to establish woodchuck HCC as a model for image-guided interventional oncology therapies, novel drug development, and investigation of immune effects.

\section{Methods}

\section{Animal Procedures}

This study was conducted under an animal use protocol approved by the National Institutes of Health Clinical Center Animal Care and Use Committee in compliance with the US Animal Welfare Regulations. Twelve woodchucks with HCC (2.45-4.9 kg; 17-24 months old; 5 females, 7 males) (Northeastern Wildlife, Harrison, Idaho, USA) were studied. The animals had been infected with woodchuck hepatitis virus (WHV) (cWHV7P2a inoculum, approximately $10^{9}$ viral particles, administered subcutaneously as newborns), ${ }^{14}$ and were confirmed as tumor-positive by the vendor, based on measurement of gamma-glutamyl transferase liver enzyme where levels above $50 \mathrm{IU} / \mathrm{L}$ would indicate $\mathrm{HCC}^{15}$ and/or diagnostic ultrasound. Of the twelve, six had been used in a proprietary drug study prior to acquisition, while six were naïve, having received no interventions. All animals were used for the radiology-pathology correlations but only the naïve animals were included in the immunohistochemistry (IHC) studies. Animals were individually housed with 12-hour light: dark cycling and ad libitum access to food and water.

\section{Imaging}

Animals were sedated and underwent multi-phasic CT imaging (Philips Brilliance MX8000 IDT 16-section Detector CT; Philips, Cleveland, OH, USA) as previously described. ${ }^{9}$ Briefly, vascular access was achieved with jugular or hindlimb cutdown and 18-G or 21-G angiocatheter insertion or insertion of a 21-G or $23-\mathrm{G}$ angiocatheter into a foreleg vein. Following noncontrast $\mathrm{CT}$, multiphase contrast imaging was performed with power injection (MEDRAD Stellant CT Injection System, Bayer Healthcare, Leverkusen, Germany) of $3.0 \mathrm{~mL}$ of iopamidol i.v. (Isovue-370, Bracco Diagnostics, Monroe Township, NJ) followed by $3.0 \mathrm{~mL} 0.9 \%$ saline, all at $0.2 \mathrm{~mL} / \mathrm{sec}$. The imaging protocol was initiated once contrast appeared in the distal thoracic aorta with acquisition in the early arterial (4-sec delay), late arterial (23-sec delay), portal venous 
(43-sec delay), late parenchymal (63 sec), and delayed (2 min) phases. Nine animals underwent ultrasound imaging (Philips iU22, Philips, Bothell, WA) with linear (5-12 MHz) and convex (2-5 MHz) probes, including color Doppler. Six animals underwent MRI in a clinical scanner (Achieva 3.0T, Philips, Cleveland, $\mathrm{OH}$ ). Using a standard 32-ch cardiac RF receive coil, MRI was performed in the axial plane with slice thickness of 1.5-3.0 mm, and field of view 120-192 $\mathrm{mm}$. The following sequences were acquired: T1-weighted HighResolution Isotropic Volume Examination (THRIVE), T2-weighted Turbo Spin Echo (TSE), and diffusionweighted imaging (DWI). Contrast enhanced T1 images were acquired after administration of gadoteridol, 0.1 $\mathrm{mmol} / \mathrm{kg}(0.2 \mathrm{~mL} / \mathrm{kg})$ (Prohance, Bracco Diagnostics, Monroe Township, NJ), diluted in $0.9 \%$ saline to a total volume of $3.0 \mathrm{~mL}$ and delivered by power injection (MEDRAD) followed by $3.0 \mathrm{~mL} 0.9 \%$ saline, all at $0.2 \mathrm{~mL} / \mathrm{sec}$. These pulse sequences were selected to represent typical sequences used in clinical MRI liver examinations, both with and without fat suppression. At the conclusion of the studies, animals were euthanized by intravenous administration of Beuthanasia-D, $1 \mathrm{~mL} /$ $10 \mathrm{lbs}$ (pentobarbital sodium $390 \mathrm{mg} / \mathrm{mL}$ and phenytoin sodium $50 \mathrm{mg} / \mathrm{mL}$; Schering-Plough, Kenilworth, NJ).

\section{Radiological Analysis}

All CT, MRI and US images were transferred to a workstation and image analysis was performed by a boardcertified radiologist. The following features obtained at CT and MRI were evaluated for each detected tumor:

1. Diameter of tumor: three dimensions were measured on images that provided the best delineation of tumor margins;

2. Enhancement pattern at the arterial phase;

3. Washout appearance during the venous and delayed phases;

4. Capsular enhancement; and

5. Tumor invasion into vasculature.

Ancillary features such as corona enhancement, mosaic architecture, nodule in nodule appearance, restricted diffusion, T2 hyperintensity, and intratumoral fat were also noted. All hepatic tumors detected on CT and MRI were assigned a LI-RADS score using the 2018 algorithm. ${ }^{16}$

\section{Tissues Collection, Processing, and Radiology-Pathology Correlation}

For seven woodchucks, harvested tissues were sectioned manually at approximately $10 \mathrm{~mm}$ intervals (Figure 1B) and placed directly into $10 \%$ neutral buffered formalin. Multiplanar reconstruction of CT images was performed to match the images with the plane of the sections. In order to achieve a more accurate correlation between the imaging features and histology, tissue from the remaining five animals were sectioned using tumor-specific, 3-D-printed sectioning molds based on segmentation of in-vivo CT or MRI as previously described (Figure 1E-F) ${ }^{17}$ All tissues were fixed in formalin and processed through a series of increasing concentrations of ethanol, cleared in xylenes and embedded in paraffin blocks for sectioning.

\section{Pathology and Immunohistochemistry Analysis}

Sequential $5-\mu \mathrm{m}$ thick sections from the blocks were mounted onto regular or oversized $(75 \times 50 \mathrm{~mm})$ glass slides (Fisher Scientific, MA, USA). For histologic examination, one tissue section was obtained from each paraffin block and slides were stained with hematoxylin and eosin (H\&E). Since the observed histological hepatic alterations and tumors are similar to those seen in the corresponding disease in human HBV carriers and have been previously described in WHV infected animals, ${ }^{18-20}$ the same terminology was applied in the pathological analysis. Reticulin stain (Agilent Dako AR17911) was performed on a Dako Artisan automated stainer and evaluated in conjunction with $\mathrm{H} \& \mathrm{E}$ stain.

The remaining sections from each block were used for immune characterization of tumors in the 6 naïve animals for the following markers: CD3, CD4, PD-L1, the human HCC biomarker alpha-fetoprotein (AFP), FOXP3, and NCAM1. A list of all antibodies is provided in the Supplementary Table. Woodchuck spleen tissue was used as positive control. The sections used for IHC were selected to maximize the number of histologic types included. The numbers of each histologic type stained with each antibody were: normal (liver with hepatitis), $\mathrm{N}=9$ or 10 ; basophilic foci, $\mathrm{N}=3$ or 4 ; dysplasia, $\mathrm{N}=8$ or 9; and, HCC, $\mathrm{N}=13$. AFP quantification was performed on 13 normal liver tissue area, 8 premalignant foci (basophilic and dysplastic foci), and $13 \mathrm{HCC}$ tumors.

H\&E stained sections were reviewed by a veterinary pathologist. Tissues were classified as normal (i.e., no 

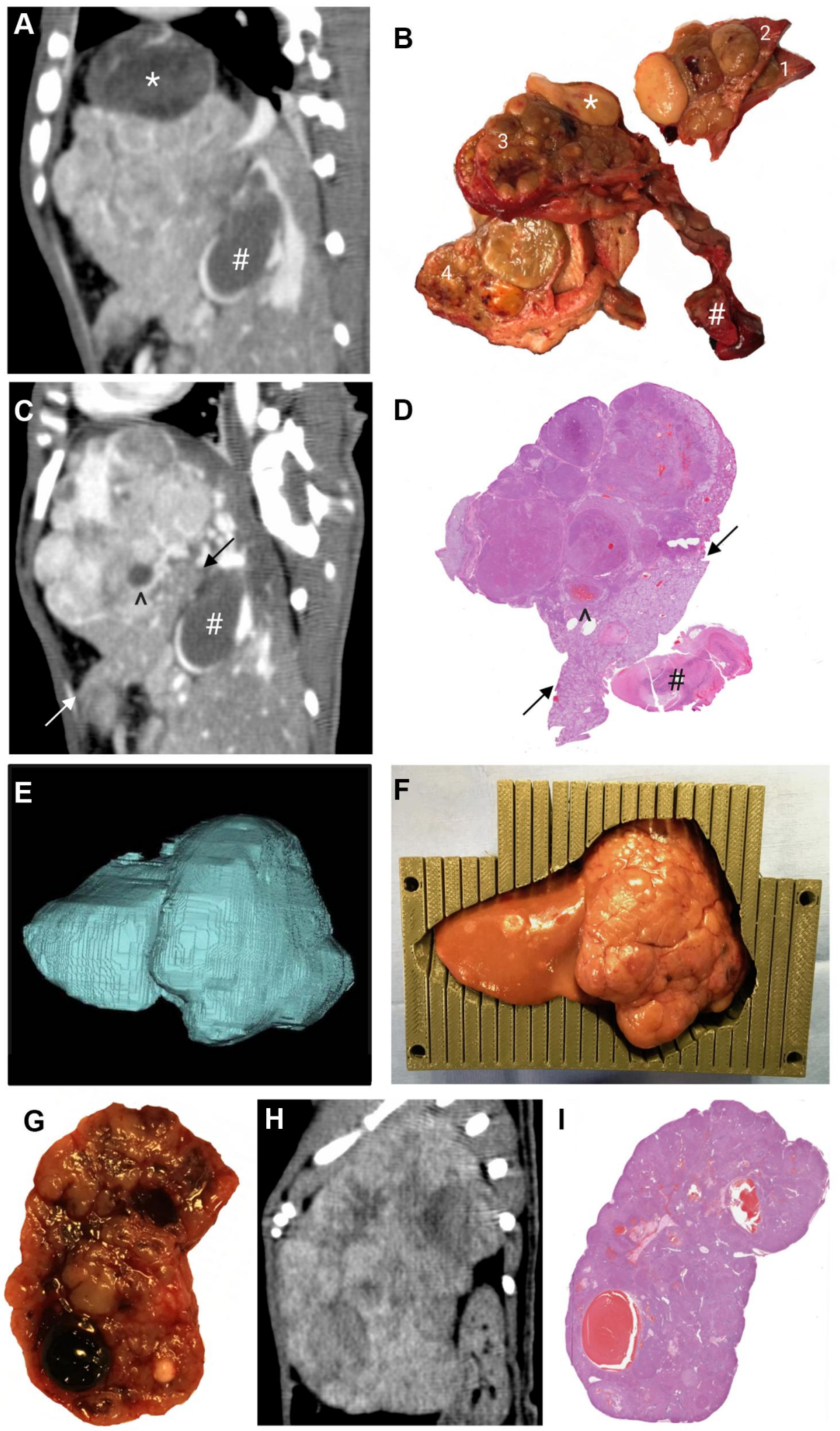

Figure I Sectioning of liver or tumor and correlation with histopathology. Tissues were sectioned using two methods: manual sectioning (A-D) or a 3-D sectioning mold technique for cutting (E-I). (A) In-vivo CT sagittal reconstruction in the late arterial phase of a tumor. (B) Excised whole tumor manually sectioned in the sagittal plane of the same tumor shown in (A); four sections labeled I-4. (C) Correlation between the in-vivo CT image after minor multiplanar reconstruction manipulation to correspond to the plane of the histopathology section (D) from section 3 in panel (B), matching uninvolved liver (between arrows), thrombus within tumor vasculature (^ in C, D), and occlusive portal vein thrombus (\# in A-D). The large fatty nodule $\left(^{*}\right)$ that is seen in the original sagittal CT plane (A) and in the excised whole tumor (B, section 3 ) is not identifiable in the histopathology slide. (E) Representative 3-D segmentation of an entire lobe based on in-vivo CT scan. (F) Unfixed liver lobe with tumor placed inside the 3-D sectioning mold. (G) Formalin fixed 5 mm thick tumor section obtained from the 3-D sectioning mold. (H) Corresponding sagittal CT image, late arterial phase. (I) H\&E-stained section from G corresponding to the CT image in $\mathbf{H}$. 
tumor), basophilic foci, dysplasia, adenoma, or HCC, and matched with corresponding imaging sections for correlation of radiological features and histological type.

All H\&E and IHC sections were scanned at 20X using an Aperio AT2 scanner (Leica Biosystems, Buffalo Grove, IL). Digital image analysis was performed and all histologic tumor types manually annotated by a blinded veterinary pathologist. Each entire section was annotated with histologic types. Areas of artifact such as folds and tears were excluded from analysis. For each IHC stain, DAB (3,3'Diaminobenzidine) was performed. Image analysis was performed using Cytoplasmic V2 algorithm in ImageScope version 12.4. AFP H-score (ranging from 0 to 300) was calculated automatically by multiplying the percentage of positive cells by the staining intensity. For immune infiltrates the percentage of positive pixels in the region of interest was determined and used as a surrogate for determining positive cell number and positive cell percentage as depicted by the nuclear stain. In order to investigate immune infiltrates in the tumor microenvironment along the HCC carcinogenesis axis, the presence and distribution of immune cells within each histological type were compared to surrounding liver parenchyma.

\section{Statistical Analysis}

For each imaging characteristic, sensitivity, the proportion of malignant tumors that were determined to be malignant (HCC and dysplasia), and specificity, the proportion of tumors that were determined to be benign (basophilic foci, adenoma, lipidosis foci), were calculated. Statistical analyses were performed using GraphPad Prism 8.4.2 (GraphPad Software, San Diego, California).

Repeated-measures models were used to account for the correlation between AFP or immune cell count in IHC taken from the same animal. The dependent variable was defined as stain count and the independent variable was histological type. Stain counts between different pairs of histological types were compared based on $t$-tests applied to differences in least-squares means obtained from the model. Because all reported p-values were unadjusted for multiple comparisons and using the general Bonferroni rule of thumb, p-values less than $0.005(\approx 0.05 / 10$ tests $)$ can be considered as reflecting evidence of a difference, where 10 is the number of tests of interest. Analyses were done with SAS Version 9.4 software (SAS Institute, Inc., Cary, North Carolina).

\section{Results}

\section{Imaging Characteristics and Radiology-Pathology Correlation}

Forty tumors were detected on CT or MRI and were analyzed for radiology-pathology correlation. In vivo tumor images were matched with manually sectioned tumor and liver pathology sections (Figure 1A-D). However, the use of CT-based 3-D sectioning molds facilitated a more accurate and expeditious correlation between in vivo tumor imaging features and liver pathology sections without imaging adjustments (Figure 1E-I).

US, multi-phasic CT, and MRI demonstrated tumors of varying size, vascularity, enhancement, and heterogenicity (Figures 2 and 3A -B). On US imaging, tumors were depicted with heterogeneous echogenicity and nodular appearance (Figure 2A-B). A linear probe was the most sensitive modality with numerous foci smaller than $1 \mathrm{~cm}$ found scattered throughout the liver (Figure 2C) in some animals. Doppler US demonstrated the robust arterial blood supply to the tumors as well as large caliber arteriovenous shunts (Figure 2D-F). On CT, the majority of detected tumors regardless of size and across different histology types showed early arterial enhancement which persisted into the late arterial phase. Wash-out in the portal venous phase or the delayed phase was detected in only 13 out of $29 \mathrm{HCC}$ tumors (Figure 3A). Twelve HCC tumors presented with heterogeneous and nodular appearance on imaging. This feature was present only in tumors that were confirmed to be HCC on pathological analysis as opposed to homogeneous tumors which were associated with dysplastic or basophilic foci histology. Lipid depositions, calcifications, and distinct nodules contributed to this heterogenous appearance (Figure 3A). On MRI, the most sensitive sequence in which malignancy could be detected was DWI. All HCC tumors in the six animals had high signal intensity on DWI while benign tumors did not. One of 2 dysplastic foci presented with high DWI signal.

Detailed description of the radiology-pathology correlation that could be achieved with the 3-D sectioning mold technique using MRI analysis is presented in Figure 3B and C. Segmentation of the tumor in the DWI sequence revealed that it was only part of the high signal of the tumor in the THRIVE T1 sequence. Analysis of the corresponding H\&E slide revealed that only the region with high DWI signal was $\mathrm{HCC}$ while the adjacent region was benign basophilic focus.

Human LI-RADS scoring was applied to all tumors based on CT and MRI. LI-RADS scoring was as follows: 26 of 29 HCC tumors were categorized as LR-4 or 5, 3 of 3 dysplastic 

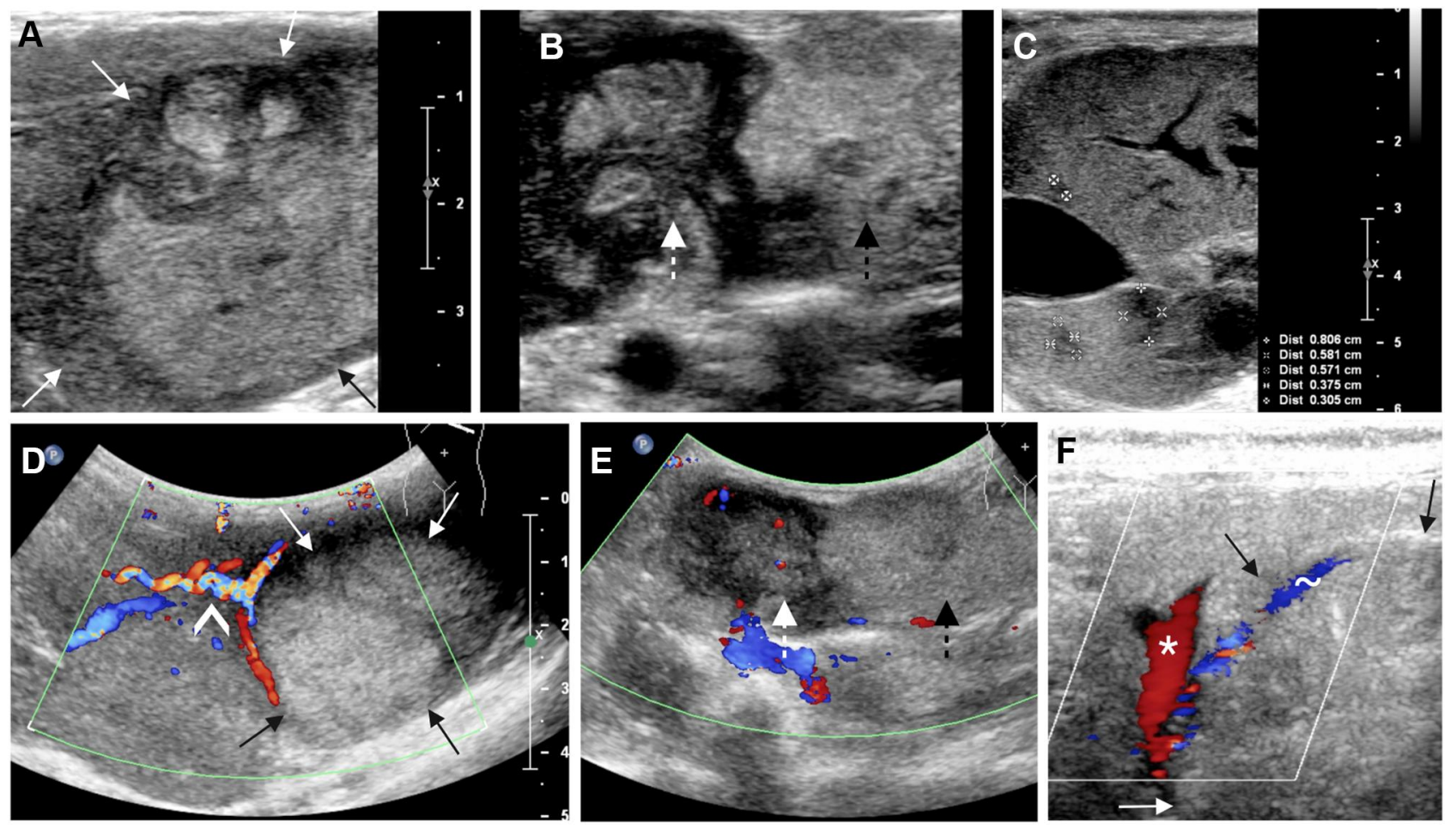

Figure 2 US characterization of woodchuck hepatic tumors. US images acquired with a linear probe (A-C). (A) Tumor (within arrows) demonstrating heterogenous echogenicity, mostly hyper-echogenicity with lobulated margins. (B) Additional example of pedunculated and multinodular tumor that demonstrates heterogeneous echogenicity in different nodules of the same tumor (dashed arrow). (C). Numerous small hypoechoic nodules scattered within the left lateral lobe of the liver (within the size markers). Color doppler images (D-F). (D) The tumor within arrows is the same tumor and plane as in $(\mathbf{A})$, large corkscrew vessel supplies the tumor $\left({ }^{\wedge}\right)$. (E) Same tumor and plane as in (B). Tumor heterogeneity was depicted not only in texture and echogenicity but also in vascularity, a hypoechoic region (white arrow) is more vascular than the hyperechoic region (black arrow). (F) Color Doppler ultrasound of a tumor (within arrows) shows a large draining vessel ( ) and its confluence with the portal vein $(*)$ representing a tumoral arterioportal shunt.

foci were categorized as LR-4 or 5 while all eight benign tumors, basophilic foci, adenoma, and lipidosis foci, were categorized as LR-3 (Table 1). Tumor size larger than $2 \mathrm{~cm}$, with heterogenous appearance on any imaging modality would be suggestive for HCC. More indicative appearance of HCC would be exophytic tumor with robust arterial blood supply.

\section{Pathology Evaluation}

Pathologic evaluation of the liver parenchyma revealed moderate to marked portal lymphocytic infiltrates, biliary hyperplasia and foci of vacuolated cells adjacent to portal areas, which was consistent with chronic hepatitis and was observed in all subjects. HCC diagnosis of tumors with necrosis, cystic vascular degeneration and hemorrhage was straightforward. Tumors smaller than $5 \mathrm{~mm}$ presented more challenging histopathological diagnoses. $\mathrm{H} \& \mathrm{E}$ analysis revealed numerous tumors, some of which were not detected on imaging. Reticulin stains were applied to 21 tumors (mean largest diameter $2 \mathrm{~mm}$, range $0.6-4.2 \mathrm{~mm}$ ) that were not detected on imaging. Criteria used for classification of human hepatocellular liver tumors was applied. In basophilic foci (benign tumors) the hepatic plates demonstrated trabecula composed of a single or double layer of hepatocytes. In dysplasia either focally thickened hepatocyte plates with or without a high nuclear to cytoplasmic ratio was noted. HCC tumors were characterized by widened ( 3 or more cells) hepatocyte plates and loss of reticulin (Figure 4).

\section{HCC Marker and Immune Characterization of Tumor Microenvironment}

Woodchuck tumors demonstrated positive staining for AFP, a human HCC biomarker (Figure 3D). AFP expression was heterogeneous in tumors; however, HCC tumors did not express higher levels of AFP compared to normal parenchyma with hepatitis or premalignant tumors (Figure 3E, $\mathrm{P}=0.53$ ).

The immune cells investigated and the specific IHC stains included $\mathrm{T}$ cells (CD3), $\mathrm{T}$ helper cells (CD4), natural killer cells (NCAM), T regulatory cells (FOXP3), and PD-L1 (Figure 5). No differences in the quantity of immune cells 

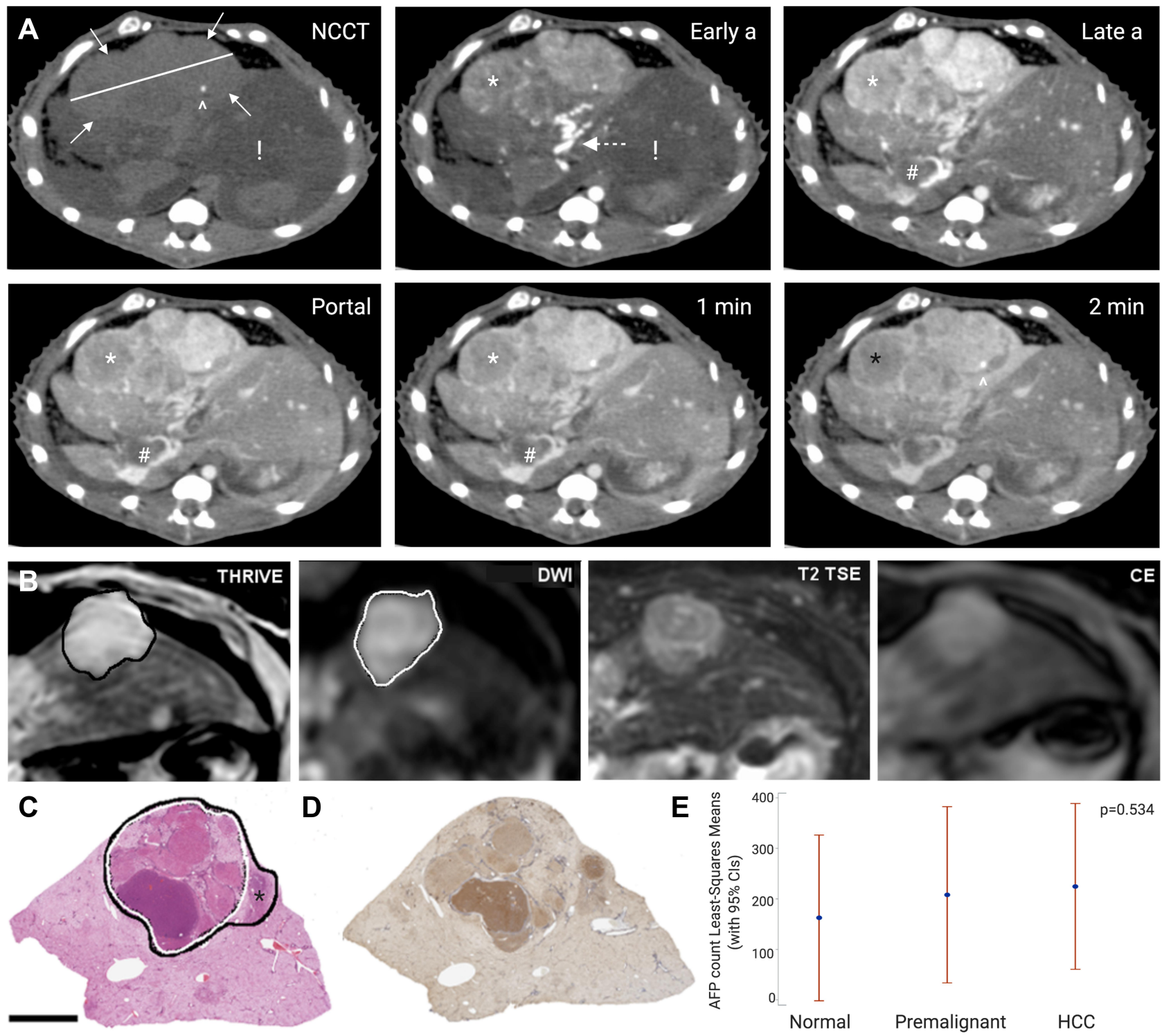

$\mathrm{p}=0.534$

Figure 3 Tumoral CT, MRI, and histological correlation. (A) Computed tomography (CT) axial images of the woodchuck liver tumor that is presented in Figure IA-D. All panels are at the same level in different phases of contrast administration: Non-contrast CT (NCCT), early arterial (Early a), late arterial (Late a), portal venous phase, I minute, and 2 minutes. This large tumor, $4.8 \mathrm{~cm}$ in largest dimension (tumor measurement on NCCT panel), replaced the entire right lateral and quadrate lobes and was characterized by a nodular appearance, enhancement in the early arterial and late arterial phases, washout in the portal venous phase $(*)$, portal thrombus (\#), and a focus of calcification $\left({ }^{\wedge}\right)$. The background liver demonstrates a hypodense appearance (!) that correlated with steatosis and inflammation which is present in woodchucks chronically infected with WHV. A robust arterial blood supply by a corkscrew artery is demonstrated in the early arterial phase (dashed arrow). (B) Magnetic resonance imaging (MRI) characterization of a $1.6 \mathrm{~cm}$ tumor. THRIVE TI showed the tumor with increased signal (within the black line). Diffusion weighted imaging (DWI) showed a high signal for most of the tumor (within the white line). The tumor had high signal on T2, and brightly enhanced on contrast-enhanced (CE) imaging. The tumor was sectioned using the 3-D mold technique. (C) H\&E stain of the corresponding tissue section with the components of the tumor outlined. The area with high DWI signal (white line) was confirmed as HCC while the part of the lesion that did not show high DWI signal $(*)$ was benign with a basophilic focus histology. Immunohistochemistry of sequential section from the same tissue block demonstrated heterogenous alpha feto protein (AFP) stain (D). (E) Quantification of AFP shows high expression in parenchyma with hepatitis (normal), premalignant and HCC tumors. AFP expression did not differ in the different histological types.

were found between different histopathology types with one exception: NCAM count was found to be 2-5 times lower in tumors relative to normal parenchyma (Figure 5, row 4, $\mathrm{P}<0.001)$. Qualitative analysis suggested that immune markers were spread throughout normal liver and basophilic foci but were mainly located in the periphery of dysplastic and HCC tumors (Figure 5, columns 3 and 4). Tumors that were investigated did not express the inhibitory checkpoint protein PD-L1 (Figure 5 bottom row).

\section{Discussion}

Radiologic-pathologic correlation is an important linkage enabling more informed hypotheses in diagnosis, treatment planning, and mechanistic evaluation. Currently, commonly 
Table I Tumor Characteristics and LI-RADS Scoring for Different Tumor Histopathologies

\begin{tabular}{|l|l|l|l|l|l|}
\hline & HCC & Dysplasia & Basophilic Foci, Adenoma, Lipidosis & Sensitivity & Specificity \\
\hline Number of tumors & 29 & 3 & 8 & \\
Mean largest diameter, cm (SE) & $3.3(0.6)$ & $1.6(0.5)$ & $0.4(0.16)$ & \\
Mean volume, cm (SE) & $21.7(4)$ & $7.2(2.1)$ & $0.06(0.02)$ & & \\
Arterial hyper enhancement & $28 / 29$ & $2 / 3$ & $6 / 8$ & $94 \%$ & $25 \%$ \\
Wash-out & $13 / 29$ & $1 / 3$ & $0 / 8$ & $37 \%$ & $100 \%$ \\
Heterogeneity (mosaic) & $12 / 29$ & $0 / 3$ & $0 / 8$ & $93 \%$ & $100 \%$ \\
Restricted diffusion & $12 / 12$ & $1 / 2$ & $0 / 2$ & $61 \%$ & $100 \%$ \\
TI hyper attenuation & $6 / 11$ & $2 / 2$ & $0 / 2$ & $50 \%$ & $100 \%$ \\
T2 mild attenuation & $6 / 11$ & $0 / 1$ & $0 / 2$ & & $100 \%$ \\
LR- 3 & $3 / 29$ & $0 / 3$ & $8 / 8$ & $90 \%$ & $100 \%$ \\
LR- 4 & $5 / 29$ & $2 / 3$ & $0 / 8$ & & \\
LR- 5 & $21 / 29$ & $1 / 3$ & $0 / 8$ & & \\
\hline
\end{tabular}

used pre-clinical models of HCC typically lack imaging features that are representative of HCC. In this study, we found that human histological classification schemes appear to be suitable for the woodchuck. A total of 61 hepatic tumors were characterized and were found to generally recapitulate the histological spectrum of human hepatocellular tumors, including the HCC-specific features of reticulin distribution and AFP expression. Although histologic subtypes of woodchuck hepatocellular tumors have been reported, ${ }^{19}$ these have previously been based mainly on H\&E classification alone. Use of reticulin staining allowed better classification of early tumors along the spectrum of human carcinogenesis axis. Elevated AFP can be found in the serum of woodchucks with hepatitis or HCC, as well as seasonal elevations related to hibernation. ${ }^{21}$ We observed AFP expression in tumoral and non-tumoral liver tissues which may reflect the diffuse and ongoing liver insult related to chronic infection.

In this study, the imaging features of woodchuck hepatic tumors were described along the WHV carcinogenesis axis using imaging modalities and protocols that are typical for the management of human patients with HCC. Precise imaging and pathology correlation were enabled with liver or tumorspecific 3D-printed sectioning molds. Tumors were classified into histologic types with imaging correlations, analogous to human tumors. The most sensitive MR imaging sequence for distinguishing between malignant $\mathrm{HCC}$ or pre-malignant dysplasia and benign pathology in woodchucks was MR DWI, although the number of benign lesions included was small. This sequence enabled improved detection and better characterization of human $\mathrm{HCC}$, and is significantly correlated with human HCC histologic grade. ${ }^{22}$ Interestingly, most woodchuck tumors across all histology types expressed arterial hyperenhancement, however the wash-out imaging feature offered only $44 \%$ sensitivity. Size (largest diameter $>2 \mathrm{~cm}$ ) and mosaic appearance (tumor heterogeneity) offered high specificity for the detection of malignant tumors.

Woodchuck tumor imaging features were classified according to human LI-RADS criteria. LR-4 and LR-5 showed high sensitivity (90\%) and specificity (100\%) for the detection of malignant and pre-malignant tumors. The ability to predict pathology with standard human imaging criteria supports the value of the woodchuck model for translational inquiry. This correlation was achieved even though the majority of the animals did not have sequential imaging and thus one major criteria, growth over time, was missing. Furthermore, the lesion size criteria were not adjusted to the small animal size and relative scale compared to humans.

The immune landscape in the woodchuck liver tumor microenvironment was also characterized. The liver is a tolerogenic organ with exquisite mechanisms of immune regulation prone to an immunosuppressive tumor microenvironment, immune exhaustion, and tumor-educated regulatory T-cells. Furthermore, typical inflammation-linked carcinogenesis involves immune evasion that occurs during the initiation and evolution of HCC. ${ }^{23}$ Quantitative analysis of selected immune markers across woodchuck histology types that included tumor margins revealed no significant differences in tumoral infiltrating lymphocytes (CD3) or their subpopulation $\mathrm{T}$ helper cells (CD4), and T-regulatory cells (FOXP3) compared to the normal surrounding parenchyma. Notably, qualitative evaluation of cellular distribution revealed that $\mathrm{T}$ cells were preferentially located in the tumor margins for all histological types. Also, there was a significant decrease in natural killer cells in the tumors compared to normal 


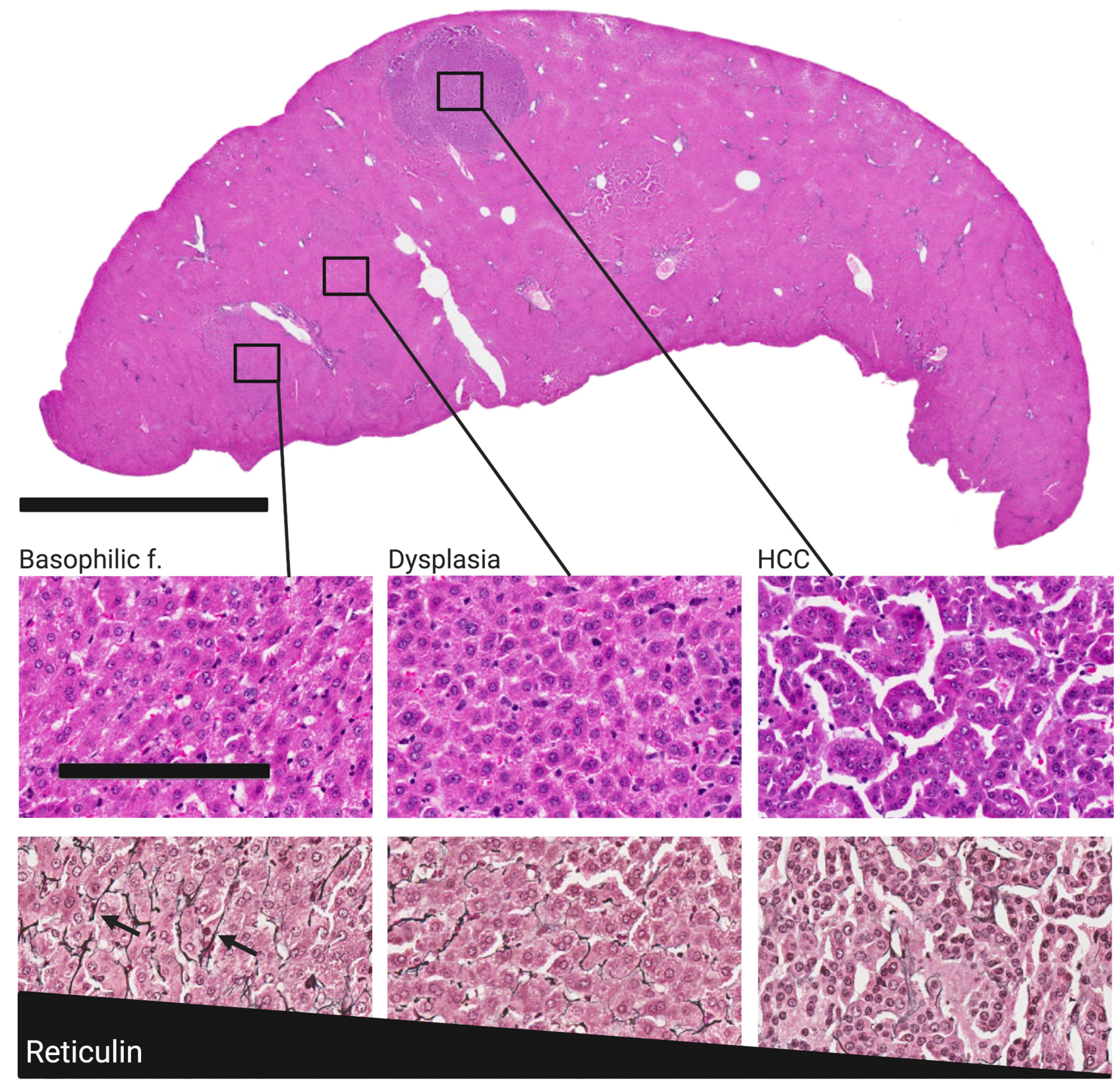

Figure $4 \mathrm{HCC}$ carcinogenesis axis in small non-imageable tumors in liver sectioned with the 3-D sectioning mold technique. The three columns from left to right are: basophilic focus, dysplasia, and HCC. Whole section H\&E stain (top) demonstrating coexistence of three different histopathologies in a single liver section, bar = 7 mm, with each shown in high magnification H\&E stained sections (top row). Reticulin stained sections (bottom row) for each demonstrate gradual loss of reticulin (arrows) from benign tumor (basophilic focus) to HCC, bar $=100 \mu \mathrm{m}$, applies to both rows.

parenchyma. The same observation has also been noted in human HCC samples. ${ }^{24}$

In human HCC, immune evasion, interplay between regulatory and cytotoxic $\mathrm{T}$ cells, ${ }^{25}$ as well as immune cell densities ${ }^{26}$ play key roles in the immune state and progression of HCC. In this study, custom processing and analysis of whole mount specimens enabled robust characterization of a spectrum of different histologies within the same animal and with wide-range spatial analysis of the entire tumor and surroundings. Establishing this large tissue bank along the carcinogenesis spectrum could allow in-depth analysis of the mutational landscape and immune characterization along this axis. This framework will potentially enable development of imaging and pathology tools such as artificial intelligence prediction models which may in turn lead to improved methods for assessment of therapy selection or targeting. 


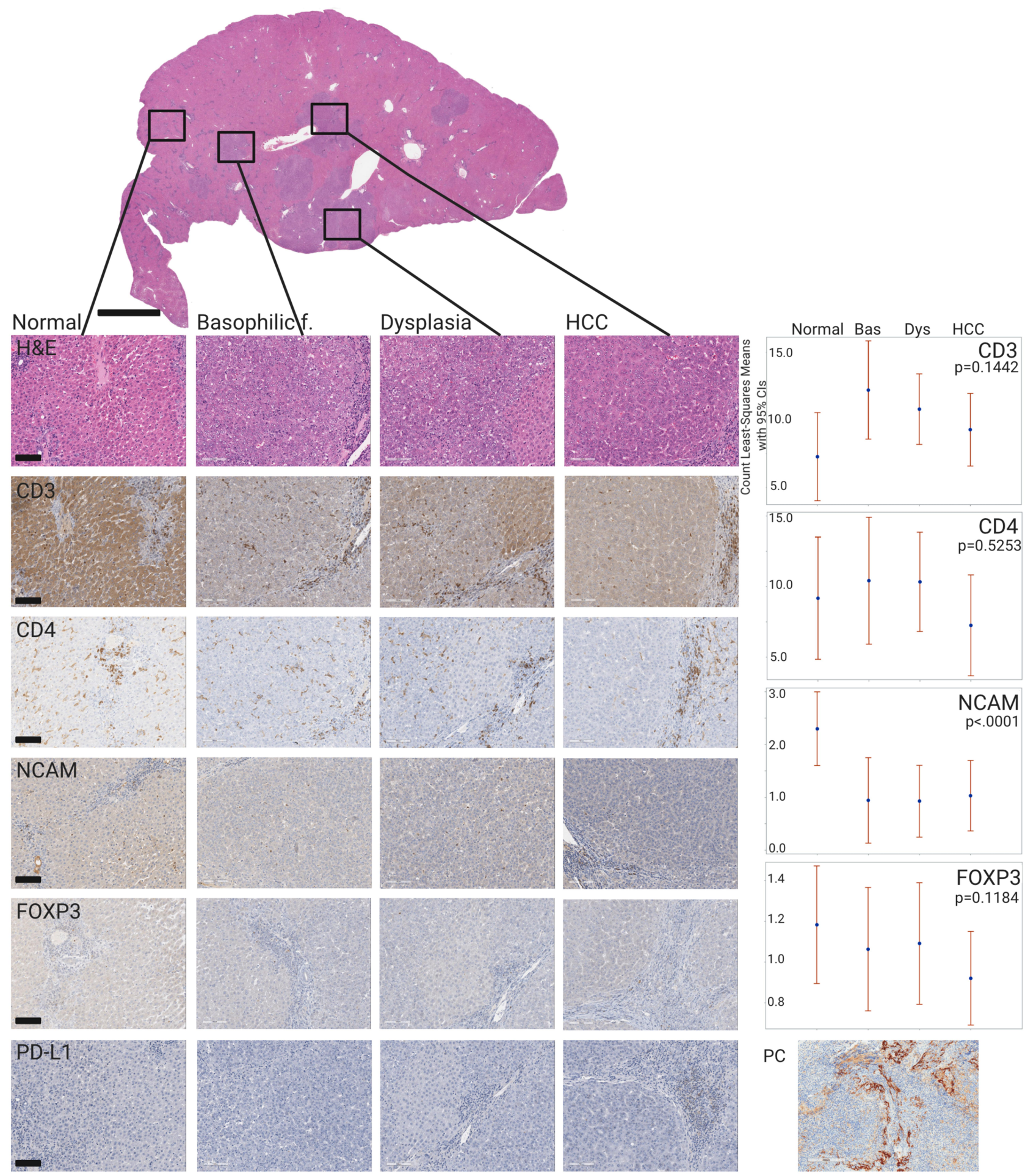

Figure 5 Immune infiltrates along HCC carcinogenesis axis in liver sectioned with the 3-D sectioning mold technique. The four columns from left to right are: normal liver, basophilic focus, dysplasia, and HCC. The top row shows representative H\&E sections demonstrating coexistence of four different histopathologies in a single liver. Subsequent rows demonstrate immunohistochemical expression of immune infiltrates by row for each tumor type as stated in the left panel of each row: CD3, CD4, NCAM (natural killers), FOXP3 (T-reg), and PD-LI for each histological type. The fifth column represents quantification of the IHC marker expression levels for each of the four histological types ( $<<0.000$ I for NCAM). PD-LI stain was negative in all histological types; a positive control (PC) stain of woodchuck spleen tissue is provided.

The two orthotopic animal models most commonly reported in the investigation of immune effects of interventional oncology tools on liver cancer are likely rabbits with implanted VX2 tumors ${ }^{27}$ and rats implanted with HCC cell lines. $^{28,29}$ The rabbit VX2 model has several major constraints. Histologically, VX2 is a squamous cell-like tumor 
induced by papillomavirus ${ }^{30}$ which does not resemble human HCC. VX2 does not recapitulate molecular processes, immune response, and angiogenic or growth factors of human $\mathrm{HCC}$ and also differs in responsiveness to antivascular agents such as bevacizumab or sorafenib. ${ }^{31}$ In addition, the VX2 model has no underlying liver disease, unlike most HCC patients. In the VX2 model, cells that have been grown in one animal are inoculated into the liver of another, which may cause confounding immune responses influencing outcomes and may hamper extrapolation of results to immune processes in human HCC. The woodchuck hepatic cancer model may be a more relevant model for interventional oncology investigations. ${ }^{9,32}$ This autochthonous tumor model may better resemble immune responses in HCC, as the tumors spontaneously develop on the carcinogenic background of WHV. This gradual sequence of events may offer a superior platform for genomic and immune investigations along the spectrum of hepatic tumors from benign to malignant. However, the shorter lifespan of the woodchuck may limit recapitulation of the full extent of chronic inflammatory fibrotic liver disease often present in humans, where HCC develops on a background of chronic hepatitis or alcoholinduced cirrhosis. The utility of the woodchuck model may still however enable collection of serial liver, tumor, and peripheral blood mononuclear cell samples at experimentally defined time points along an HCC carcinogenesis spectrum, verified by human LI-RAD criteria.

Our study had multiple additional limitations. The sample size was small and the 3D sectioning mold technique was only used for a subset of specimens. In addition, we did not have a full imaging data set that included all imaging modalities for all of our animals as well as sequential imaging over time. Characterization of multiple immune cell subpopulation expression was not feasible due to the lack of availability of well-established and validated specific antibodies for a majority of relevant markers in woodchucks. Although this gap could be addressed in the future due to recent sequencing of the full woodchuck genome, ${ }^{33}$ this report may inform future immune investigations with this unique model on the way to a broader understanding. Nevertheless, our report is limited to few major immune subpopulations. Other important immune components such as tumor-associated macrophages and B-cells are missing. Also, to be defined in future studies is whether the model can fully recapitulate the immune effects of specific immuneoncology therapies.

Woodchuck HCC may be an important biological and immunological model for human $\mathrm{HCC}$, but this model requires further validation of methodology and metrics to fully realize its translational potential. Detailed radiological, histological, and immune characterization in the woodchuck HCC model may enhance our understanding of immune responses to locoregional therapies and in turn may facilitate more reliable development of novel immune modulators to treat HCC.

\section{Abbreviations}

WHV, woodchuck hepatitis virus; HCC, hepatocellular carcinoma; CT, computed tomography; MRI, magnetic resonance imaging; LI-RADS, Liver Imaging Reporting and Data System; IHC, immunohistochemistry; H\&E, hematoxylin and eosin; AFP, Alpha-feto protein; THRIVE, T1weighted High-Resolution Isotropic Volume Examination; TSE, Turbo Spin Echo; DWI, diffusion-weighted imaging.

\section{Author Contributions}

All authors made a significant contribution to the work reported, whether that is in the conception, study design, execution, acquisition of data, analysis and interpretation, or in all these areas; took part in drafting, revising or critically reviewing the article; gave final approval of the version to be published; have agreed on the journal to which the article has been submitted; and agree to be accountable for all aspects of the work.

\section{Funding}

This work was supported by the Center for Interventional Oncology in the Intramural Research Program of the National Institutes of Health (NIH) by intramural NIH Grants NIH Z01 1ZID BC011242 and CL040015. Dr. Mauda-Havakuk is supported by the Clinical Translational Fellowship Program of the NIH Clinical Center and the Intramural Research Program of the National Institute of Biomedical Imaging and Bioengineering. NIH has a Materials Transfer Agreement with Northeastern Wildlife. The NIH and Biocompatibles UK Ltd-Boston Scientific Corporation have a Cooperative Research and Development Agreement providing support for this research. NIH had control over the conduct of the study, the inclusion of any data, data analysis and interpretation, manuscript preparation and decisions on submission for publication. The content of this manuscript does not necessarily reflect the views or policies of the US Department of Health and Human Services. The mention of commercial products, their source, or their use in connection with material reported herein is not to be construed 
as an actual or implied endorsement of such products by the United States government.

\section{Disclosure}

MTK holds equity in a human clinical trial software company, Florence Healthcare. Professor Andrew L Lewis is an employee of Biocompatibles UK Ltd, the company sponsoring the study. BJW is the Principal Investigator in the following projects: Cooperative Research \& Development Agreements between NIH and the following: BTG Biocompatibles/Boston Scientific, Siemens, Philips, NVIDIA, Celsion Corp, Canon Medical, XAct Robotics. BJW and NIH are party to Material Transfer or Collaboration Agreements with: Angiodynamics, 3T Technologies, Profound Medical, Exact Imaging, Johnson and Johnson, Endocare/Healthtronics, and Medtronic. Outside the submitted work, BJW is primary inventor on 41 issued patents owned by the NIH (list available upon request), a portion of which have been licensed by NIH to Philips. BJW and NIH report a licensing agreement with Canon Medical on algorithm software with no patent. BJW is joint inventor (assigned to HHS NIH US Government (for patents and pending patents related to drug eluting bead technology, some of which may have joint inventorships with BTG Biocompatibles/ Boston Scientific. BJW is primary inventor on patents owned by $\mathrm{NIH}$ in the space of drug eluting embolic beads. The authors report no other conflicts of interest in this work.

\section{References}

1. Santillan C, Fowler K, Kono Y, Chernyak V. LI-RADS major features: CT, MRI with extracellular agents, and MRI with hepatobiliary agents. Abdom Radiol (NY). 2018;43:75-81. doi:10.1007/s00261-0171291-4

2. European Association For The Study Of The Liver, European Organisation For Research and Treatment of C. EASL-EORTC clinical practice guidelines: management of hepatocellular carcinoma. J Hepatol. 2012;56:908-943.

3. Llovet JM, Zucman-Rossi J, Pikarsky E, et al. Hepatocellular carcinoma. Nat Rev Dis Primers. 2016;2:16018.

4. Li D, Sedano S, Allen R, Gong J, Cho M, Sharma S. Current treatment landscape for advanced hepatocellular carcinoma: patient outcomes and the impact on quality of life. Cancers (Basel). 2019;11. doi:10.3390/cancers11060841

5. Gauthier A, Ho M. Role of sorafenib in the treatment of advanced hepatocellular carcinoma: an update. Hepatol Res. 2013;43:147-154. doi:10.1111/j.1872-034X.2012.01113.x

6. Finkelmeier F, Waidmann O, Trojan J. Nivolumab for the treatment of hepatocellular carcinoma. Expert Rev Anticancer Ther. 2018;18:1169-1175. doi:10.1080/14737140.2018.1535315
7. Finn RS, Qin S, Ikeda M, et al. Atezolizumab plus bevacizumab in unresectable hepatocellular carcinoma. $N$ Engl $J$ Med. 2020;382: 1894-1905. doi:10.1056/NEJMoa1915745

8. Mestas J, Hughes CCW. Of mice and not men: differences between mouse and human immunology. J Immunol. 2004;172(5):2731-2738. doi:10.4049/jimmunol.172.5.2731

9. Pritchard WF, Woods DL, Esparza-Trujillo JA, et al. Transarterial chemoembolization in a woodchuck model of hepatocellular carcinoma. J Vasc Interv Radiol. 2020;31(5):812-819.e1. doi:10.101 6/j.jvir.2019.08.031

10. Tennant BC, Toshkov IA, Peek SF, et al. Hepatocellular carcinoma in the woodchuck model of hepatitis B virus infection. Gastroenterology. 2004;127(5):S283-S293. doi:10.1053/j.gastro. 2004.09.043

11. Menne S, Cote PJ. The woodchuck as an animal model for pathogenesis and therapy of chronic hepatitis B virus infection. World J Gastroenterol. 2007;13:104-124. doi:10.3748/wjg.v13.i1.104

12. Putzer BM, Stiewe T, Rodicker F, et al. Large nontransplanted hepatocellular carcinoma in woodchucks: treatment with adenovirus-mediated delivery of interleukin $12 / \mathrm{B} 7.1$ genes. $J$ Natl Cancer Inst. 2001;93:472-479. doi:10.1093/jnci/93.6.472

13. Rodriguez-Madoz JR, Liu KH, Quetglas JI, et al. Semliki forest virus expressing interleukin-12 induces antiviral and antitumoral responses in woodchucks with chronic viral hepatitis and hepatocellular carcinoma. J Virol. 2009;83:12266-12278. doi:10.1128/JVI.01597-09

14. Fletcher SP, Chin DJ, Gruenbaum L, et al. Intrahepatic transcriptional signature associated with response to interferon-alpha treatment in the woodchuck model of chronic hepatitis B. PLoS Pathog. 2015;11: e1005103. doi:10.1371/journal.ppat.1005103

15. Jacob JR, Sterczer A, Toshkov IA, et al. Integration of woodchuck hepatitis and N-myc rearrangement determine size and histologic grade of hepatic tumors. Hepatology. 2004;39:1008-1016. doi:10.1002/hep.20106

16. Cerny M, Chernyak V, Olivié D, et al. LI-RADS version 2018 ancillary features at MRI. RadioGraphics. 2018;38:1973-2001. doi:10.1148/rg.2018180052

17. Mikhail AS, Mauda-Havakuk M, Partanen A, Karanian JW, Pritchard WF, Wood BJ. Liver-specific 3D sectioning molds for correlating in vivo CT and MRI with tumor histopathology in woodchucks (marmota monax). PLoS One. 2020;15. doi:10.1371/journal. pone. 0230794

18. Popper H, Roth L, Purcell RH, Tennant BC, Gerin JL. Hepatocarcinogenicity of the woodchuck hepatitis-virus. Proc Natl Acad Sci U S A. 1987;84:866-870. doi:10.1073/pnas.84.3.866

19. Roth L, King JM, Hornbuckle WE, Harvey HJ, Tennant BC. Chronic hepatitis and hepatocellular carcinoma associated with persistent woodchuck hepatitis virus infection. Vet Pathol. 1985;22:338-343. doi:10.1177/030098588502200407

20. Meng Z, Ma Z, Zhang E, et al. Novel Woodchuck Hepatitis Virus (WHV) transgene mouse models show sex-dependent WHV replicative activity and development of spontaneous immune responses to WHV proteins. J Virol. 2014;88:1573-1581. doi:10.1128/JVI.0208613

21. Cote PJ, Gerin JL, Tennant BC. a-fetoprotein in the woodchuck model of hepadnavirus infection and disease: immunochemical analysis of woodchuck a-fetoprotein and measurement in serum by quantitative monoclonal radioimmunoassay. Hepatology. 1990;11:824-833. doi:10.1002/hep.1840110518

22. Jiang HY, Chen J, Xia CC, Cao LK, Duan T, Song B. Noninvasive imaging of hepatocellular carcinoma: from diagnosis to prognosis. World J Gastroenterol. 2018;24:2348-2362. doi:10.3748/wjg.v24. i22.2348

23. Shlomai A, de Jong YP, Rice CM. Virus associated malignancies: the role of viral hepatitis in hepatocellular carcinoma. Semin Cancer Biol. 2014;26:78-88. doi:10.1016/j.semcancer.2014.01.004 
24. Wu Y, Kuang DM, Pan WD, et al. Monocyte/macrophage-elicited natural killer cell dysfunction in hepatocellular carcinoma is mediated by CD48/2B4 interactions. Hepatology. 2013;57:1107-1116. doi: $10.1002 /$ hep. 26192

25. Gao Q, Qiu SJ, Fan J, et al. Intratumoral balance of regulatory and cytotoxic T cells is associated with prognosis of hepatocellular carcinoma after resection. J Clin Oncol. 2007;25:2586-2593. doi:10.1200/ JCO.2006.09.4565

26. Gabrielson $\mathrm{A}$, Wu $\mathrm{Y}$, Wang $\mathrm{H}$, et al. Intratumoral $\mathrm{CD} 3$ and $\mathrm{CD} 8$ T-cell densities associated with relapse-free survival in HCC. Cancer Immunol Res. 2016;4:419-430. doi:10.1158/2326-6066.CIR-15-0110

27. Duan XH, Li TF, Zhou GF, et al. Transcatheter arterial embolization combined with radiofrequency ablation activates CD8(+) T-cell infiltration surrounding residual tumors in the rabbit VX2 liver tumors. Onco Targets Ther. 2016;9:2835-2844. doi:10.2147/OTT.S95973

28. Avritscher R, Jo N, Polak U, et al. Hepatic arterial bland embolization increases Th17 cell infiltration in a syngeneic rat model of hepatocellular carcinoma. Cardiovasc Intervent Radiol. 2020;43:311-321. doi:10.1007/s00270-019-02343-1

29. Ueshima E, Nishiofuku H, Takaki H, et al. Hepatic artery embolization induces the local overexpression of transforming growth factor betal in a rat hepatoma model. Liver Cancer. 2020;9:63-72. doi:10.1159/000502774
30. Schlageter M, Terracciano LM, D'Angelo S, Sorrentino P. Histopathology of hepatocellular carcinoma. World J Gastroenterol. 2014;20:15955-15964. doi:10.3748/wjg.v20.i43.15955

31. Nass N, Streit S, Wybranski C, et al. Validation of VX2 as a hepatocellular carcinoma model: comparison of the molecular reaction of VX2 and HepG2 tumor cells to sorafenib in vitro. Anticancer Res. 2017;37:87-93. doi:10.21873/anticanres.11293

32. Burke CT, Cullen JM, State A, et al. Development of an animal model for radiofrequency ablation of primary, virally induced hepatocellular carcinoma in the woodchuck. J Vasc Interv Radiol. 2011;22:1613-1618 e1611. doi:10.1016/j.jvir.2011.08.020

33. Alioto TS, Cruz F, Gomez-Garrido J, et al. The genome sequence of the eastern woodchuck (marmota monax) - a preclinical animal model for chronic hepatitis B. G3 (Bethesda). 2019;9:3943-3952. doi: $10.1534 / \mathrm{g} 3.119 .400413$

\section{Publish your work in this journal}

The Journal of Hepatocellular Carcinoma is an international, peerreviewed, open access journal that offers a platform for the dissemination and study of clinical, translational and basic research findings in this rapidly developing field. Development in areas including, but not limited to, epidemiology, vaccination, hepatitis therapy, pathology and molecular tumor classification and prognostication are all considered for publication. The manuscript management system is completely online and includes a very quick and fair peer-review system, which is all easy to use. Visit http://www.dovepress.com/ testimonials.php to read real quotes from published authors. 